EDITORIAL

\title{
Quantifying myocardial perfusion using contrast echocardiography
}

L Galiuto

\section{There is a complex relation between what can be seen using perfusion imaging techniques, and what can be measured}

Correspondence to: Dr Leonarda Galiuto, Institute of Cardiology, Catholic University of the Sacred Heart, Policlinico A. Gemelli, Largo A. Gemelli, 8, 00136 Rome Italy; Igaliuto@rm.unicatt.it
S cientific research proceeds and develops, formulating questions directed to the measurable part of the studied phenomenon thus requiring quantitative answers. Science asks for numbers as the main part of its method, although in nature there is much more than what numbers can measure! This is the case of perfusion imaging techniques where there is a complex relation between what can be seen and what can be measured. In fact, a technique can be considered clinically useful not only when it is able to provide easy visual assessment of the studied phenomenon, but also when reliable and meaningful data can be generated. However, the process of development requires the parallel improvement of the display and quantitation aspects of the technique. For myocardial contrast echocardiography (MCE), the process of development has been particularly long since the display techniques have been hampered by several pitfalls, thus generating unreliable quantitative information.

The paper by Yamada and colleagues ${ }^{1}$ in this issue of Heart focuses on the importance of "reliable" quantification of MCE. In fact, the authors begin calibrating in vitro the contrast signal intensity generated by microbubbles of Levovist within the insonified ultrasound field, thus confirming an excellent linear correlation between bubble concentration and contrast signal intensity obtained by harmonic power Doppler. Then the authors developed a method to quantitate the contrast intensity in vivo, compensating for the spatial heterogeneity of the signal among different myocardial walls generated by the acoustic field inhomogeneity as well as by distal wall contrast attenuation. Finally, they verified the clinical meaning of the generated numbers comparing normal segments with segments with perfusion defects at single photon emission computed tomography (SPECT).

\section{INTERMITTENT VERSUS REAL TIME QUANTITATIVE MCE}

In the last decade, major advances in the MCE technique have been made, moving from first to second generation bubbles with a relative improvement in the signal to noise ratio, along with the development of more sophisticated
Heart 2005;91:133-135. doi: 10.1136/hrt.2004.043760

ultrasound detection methods (power modulation, pulse inversion, etc). Furthermore, real time has taken the place of intermittent MCE, thus making patient scanning much easier. In this scenario, Yamada and colleagues ${ }^{1}$ conducted their study using methods (intermittent imaging with first generation contrast agents) that cannot be considered cutting edge. However, the association of first generation contrast agent with harmonic power Doppler triggered mode may still find clinically valuable applications, since it has been shown that signal generated by the intermittent mode is stronger than that generated by real time MCE (20-70 v 30-45 grey scale unit), although it is more heterogeneous within left ventricular myocardial walls. ${ }^{2}$ Furthermore, Dawson and colleagues ${ }^{3}$ demon- $^{-}$ strated that high mechanical index, thus intermittent, ultraharmonic methods, show better receiver operating characteristic curves as compared to low mechanical index, real time, modes with improved accuracy in coronary artery disease detection. Thus, although it may be more troublesome to achieve adequate intermittent MCE images, they appear to be ideal for quantitation purposes, particularly in conditions in which accurate detection of signal intensity is fundamental.

\section{EXPERT EYES GENERATE CORRECT NUMBERS}

It is commonly believed that quantitative analysis of any perfusion imaging technique, and of MCE in particular, might improve the accuracy and uniformity of image interpretation. However, with the current state of the art of MCE, this appears to be only a potential value, since, given the limitations of the method, quantitation may introduce an additional and much greater error. Although expertise is required to determine a perfusion defect at MCE, distinguishing it from artefacts, an even higher level of expertise is required to generate numbers from visually assessed images. For example, there are at least two ways to quantitate perfusion in any imaging technique. One would be to position a region of interest within the affected myocardial wall to obtain any possible numerical value related to perfusion. This approach requires the identification of the "affected" wall, the selection of the dimensions of the region of interest to fit in the wall, the

Abbreviations: MCE, myocardial contrast echocardiography; SPECT, single photon emission computed tomography; TIMI, thrombolysis in myocardial infarction 
exact positioning of the region of interest within the wall, and last but not least, tracking the region of interest throughout the cardiac cycle, keeping it in the same position despite the heart movements. As a consequence, in order for this approach to generate correct numbers, expert eyes have to guide the selection of the myocardial wall to be measured, the selection of the dimensions, and the positioning and tracking of the region of interest. Thus, if an expert eye is required to identify a perfusion defect, an even more expert eye is required to provide quantitative evaluation of a perfusion defect.

The second approach would be to divide the left ventricle into a given number of segments, thus generating perfusion data from each segment. However, once several numbers are generated, the investigator then needs to decide which ones to consider originated from the perfusion defect; thus expertise is necessary not only to assess perfusion visually, recognising all the artefacts, but also to interpret correctly all the numbers generated. The concept of expert eye guided quantitation is true even for apparently less complex quantitation, such as the measurement of the length of the perfusion defect. Even in this case, the investigator needs to identify the perfusion defect, distinguishing it from artefacts, and then eventually recognise the beginning and the end of a true perfusion defect, avoiding misinterpretation generated from the incorrect evaluation of basal segment attenuation or apical bubble destruction.

\section{TECHNICAL VALUE OF QUANTITATIVE MCE EVALUATION}

The successful development of both bubble and ultrasound technology has led to the production of systems implemented with "push button" access to MCE software that, associated with the use of persistent and easily available microbubbles, have provided extremely effective imaging of myocardial contrast enhancement, thus significantly helping the widespread use of MCE. However, while important for increasing the production of accurate MCE data, the easy to use software that no longer requires particular knowledge of the physics of bubble-ultrasound interaction has reduced the awareness of the way the contrast signal is generated, together with its major limitations. In this situation, it is important to take into account the presence of significant attenuation, intramyocardial heterogeneity, and temporal variation in microbubble concentration. In the study by Yamada and colleagues, ${ }^{1}$ myocardial contrast intensity was calibrated for the adjacent left ventricular cavity to compensate for the acoustic field inhomogeneity, providing quantitative parameters of perfusion that showed much less degree of variation among myocardial segments and among individuals, and demonstrated significantly reduced values in the infarcted myocardium, as compared with SPECT. The usefulness of appropriate quantitative analysis of MCE can even be pushed one step forward, to the generation of a colour coded map of calibrated contrast intensity representing microvascular damage. In fact, Yano and colleagues, ${ }^{4}$ starting from the same concept applied by Yamada and colleagues, ${ }^{1}$ not only calibrated their MCE images for contrast signal in the adjacent left ventricular cavity, thus reducing the intersegmental contrast variability from $15.8 \mathrm{~dB}$ to $6.3 \mathrm{~dB}$, but then used these data to improve the visual assessment of perfusion further; a colour coded map was constructed that preserved all the quantitative information, thus helping to identify the spatial extent of the myocardial infarction. Another approach could be that used by Lindner and colleagues, ${ }^{5}$ that validated (versus SPECT) a method of videointensity correction for the brightest videointensity present in the same echocardiographic view. Thus, when correctly generated, interpreted, and used, quantitative information helps to enhance the visual assessment of perfusion.

\section{CLINICAL VALUE OF QUANTITATIVE MCE EVALUATION}

Quantitative analysis of MCE appears particularly useful in clinical conditions in which the diagnosis of perfusion defects is not easily obtained with visual analysis, since subtle changes in microvascular flow may occur in only part of the myocardial wall. This is the case in the detection of coronary stenosis, where myocardial flow at rest is usually normal, while it is impaired during maximal vasodilatation in the presence of haemodynamically significant stenosis. In this context, subtle changes of perfusion require accurate quantitative methods to be detected since parameters related to velocity of flow are much more sensitive than those related to blood volume, and the subendocardium is affected before the subepicardium. These changes cannot be visually appreciated, while quantitative analysis appears to be an adequate way to diagnose the presence of coronary stenosis by MCE. ${ }^{6}$

Quantitative MCE may be particularly useful to provide pathophysiological meaning to the observed perfusion abnormalities. Using intermittent harmonic imaging and Optison infusion, Andrássy and colleagues ${ }^{7}$ demonstrated $^{2}$ that relative videointensity, corrected for the brightest signal, differed significantly among post-reperfusion dysfunctioning segments, from $88 \%$ of normokinetic segments to $74 \%$ of hypokinetic segments, $61 \%$ of akinetic segments with contractile reserve, and $31 \%$ of akinetic segments without contractile reserve. The same method of videointensity correction for the brightest videointensity present in the same view has been applied by Shimoni and colleagues ${ }^{8}$ in an elegant study on the evaluation of hibernating myocardium by MCE performed with intermittent pulse inversion harmonics and incremental triggering and continuous Optison infusion. In this study, the authors demonstrated that peak myocardial videointensity (MCI) correlated with microvascular density and capillary area and inversely correlated with per cent collagen content, while the MCE index of blood velocity $(\beta)$ and flow (peak MCI $\times \beta$ ) better identified recovery of function compared with microvascular density and the use of peak MCI. In another study, ${ }^{9}$ the same group of investigators showed that MCE quantitative analysis was able to distinguish between hibernating myocardium with and without contractile reserve with a sensitivity of $90 \%$, similar to that of thallium scintigraphy (92\%) and dobutamine echocardiography $(80 \%)$, and a specificity of $63 \%$ that was even higher than thallium scintigraphy (45\%) and dobutamine echocardiography (54\%).

On the other hand, the pathophysiology of the studied clinical condition needs to be taken into account in order to give the correct interpretation of perfusion maps. In fact, if the overcalling of visually assessed perfusion defects significantly impairs the specificity of MCE in the early detection of myocardial infarction (going as low as 50\% in the study of Moir and colleagues ${ }^{10}$ ), at the same time it is important to remember that recognising the presence of a perfusion defect distal to a TIMI 3 recanalised coronary artery does not necessarily mean that MCE visual analysis did not enable discrimination between post-infarct impaired and normal flow; this is because a microvascular perfusion defect in a myocardial region served by a TIMI 3 infarct related artery is exactly what has been defined and largely studied as the "no reflow phenomenon". It is important to note that in the same study, quantitative analysis of appropriate parameters of microvascular perfusion at MCE allowed territories subtended by an artery with impaired (TIMI 0-2) flow to be differentiated from those with preserved (TIMI 3 ) flow. Once 
again, numbers give correct answers when the question is formulated correctly and images appropriately guide the analysis.

Similar to nuclear or positron emission tomographic studies, an additional value of MCE quantitation can be recognised in the generation of threshold values able to provide clinically valuable information. For example, a cut off of $50 \%$ relative videointensity showed an $82 \%$ predictive value of contractile recovery either spontaneously or after dobutamine. $^{7}$

Lastly, a quantitative analysis is necessary to assess the results of a pharmacological intervention able to produce changes of microvascular flow either with therapeutic ${ }^{11}$ or diagnostic ${ }^{2}$ purposes.

The type of quantitation of MCE images relates to the type of information needed and, therefore, it is necessarily different in differing clinical conditions. In fact, while the assessment and evaluation of coronary stenosis relies on sophisticated methods of myocardial blood flow quantitation, ${ }^{12}$ in the setting of acute myocardial infarction it appears more important to quantitate the longitudinal and transmural extent of perfusion defect, while intramyocardial blood flow of the infarct zone is necessarily reduced to the level to be visually assessed. ${ }^{13}$ Although quantitative MCE evaluation has strong technical and clinical value, even with the use of more sophisticated imaging methods such as magnetic resonance imaging, it has been recently shown that visual analysis appears to be sufficient for the assessment of transmurality of myocardial infarction. ${ }^{14}$ Thus, eyes can do a great job providing good clinical answers from MCE images.

\section{REFERENCES}

1 Yamada S, Komuro K, Mikami T, et al. Novel quantitative assessment of myocardial perfusion by harmonic power Doppler imaging during myocardial contrast echocardiography. Heart 2005;91:183-8.
2 Murthy TH, Li P, Locvicchio E, et al. Real-time myocardial blood flow imaging in normal human beings with the use of myocardial contrast echocardiography. J Am Soc Echocardiogr 2001;14:698-705

3 Dawson D, Rinkevich D, Belcik T, et al. Measurement of myocardial blood flow velocity reserve with myocardial contrast echocardiography in patients with suspected coronary artery disease: comparison with quantitative gated technetium $99 \mathrm{~m}$ sestamibi single photon emission computer tomography. J Am Soc Echocardiogr 2003; 16:1171-7.

4 Yano A, Ito $\mathrm{H}$, Iwakura $\mathrm{K}$, et al. Myocardial contrast echocardiography with a new calibration method can estimate myocardial viability in patients with myocardial infarction. J Am Coll Cardiol 2004;43:1799-806.

5 Lindner JR, Villanueva FS, Dent JM, et al. Assessment of resting perfusion with myocardial contrast echocardiography: theoretical and practical considerations. Am Heart J 2000;139:231-40.

6 Fukuda S, Muro T, Hozumi T, et al. Changes in transmural distribution of myocardial perfusion assessed by quantitative intravenous myocardial contrast echocardiography in humans. Heart 2002;88:368-72.

7 Andrássy $P$, Zielinska M, Busch R, et al. Myocardial blood volume and the amount of viable myocardium early after mechanical reperfusion of acute myocardial infarction: prospective study using venous contrast echocardiography. Heart 2002;87:350-5.

8 Shimoni S, Frangogiannis NG, Aggeli CJ, et al. Microvascular structural correlates of myocardial contrast echocardiography in patients with coronary artery disease and left ventricular dysfunction. Implications for the assessment of myocardial hibernation. Circulation 2002;106:950-6.

9 Shimoni S, Frangogiannis NG, Aggeli CJ, et al. Identification of hibernating myocardium with quantitative intravenous myocardial contrast echocardiography. Comparison with dobutamine echocardiography and thallium-201 scintigraphy. Circulation 2003;107:538-44.

10 Moir S, Haluska B, Leung D, et al. Quantitative myocardial contrast echocardiography for prediction of thrombolysis in myocardial infarction flow in acute myocardial infarction. Am J Cardiol 2004;93:1212-17.

11 Galiuto L, DeMaria AN, del Balzo U, et al. Ischemia-reperfusion injury at the microvascular level: treatment by endothelin-A selective antagonist and evaluation by myocardial contrast echocardiography. Circulation 2000;102:31 11-16

12 Wie K, Jayaweera AR, Firoozan S, et al. Quantification of myocardial blood flow with ultrasound-induced destruction of microbubbles administered as a constant venous infusion. Circulation 1998:97:473-83.

13 Galiuto L, Belloni F, Raimondi F, et al. Longitudinal, transmural and intramyocardial quantitation of post-infarct microvascular damage using a novel myocardial contrast echocardiographic technology [abstract]. Eur Heart J 2004; (suppl).

14 Schiif JD, Kaandorp TAM, Lamb HJ, et al. Quantification of myocardial infarct size and transmurality by contrast-enhanced magnetic resonance imaging in men. Am J Cardiol 2004;94:284-8.

\section{IMAGES IN CARDIOLOGY}

doi: $10.1136 /$ hrt.2004.041558

\section{Löffler's endomyocarditis}

A 65 year old woman with chronic eosinophilic pneumonia was transferred to our hospital with acute dyspnoea. Physical examinations revealed signs of congestive heart failure. Blood analysis revealed an increase in eosinophil counts $\left(1547 / \mathrm{mm}^{3}\right)$. Transthoracic echocardiography showed large masses in the left ventricular apex extending to the posterior mitral leaflet with normal ventricular size and function (panel A). The motion of the posterior mitral leaflet was restricted and severe mitral regurgitation was noted (panel B). Mild tricuspid regurgitation was found and estimated right ventricular systolic pressure was $80 \mathrm{~mm} \mathrm{Hg}$. Enhanced computed tomography and magnetic resonance imaging of the heart identified the masses as mural thrombi, making the diagnosis of Löffler's endomyocarditis. She was treated with steroids, diuretics, and anticoagulation resulting in resolution of symptoms. Repeated echocardiography one month after showed decreased apical thrombi (panel C), but severe mitral regurgitation still existed.
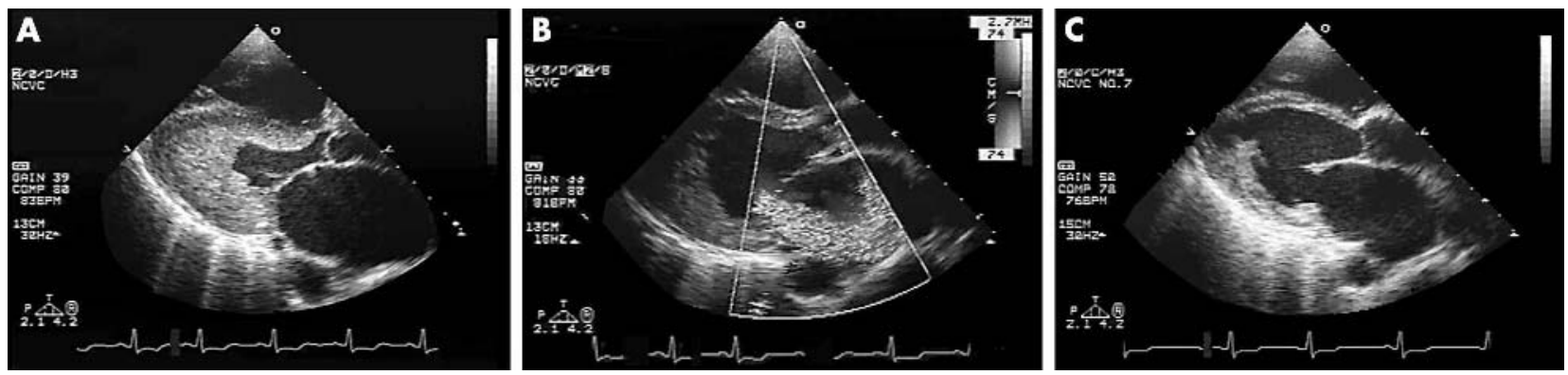

Yamamoto

S Nakatani

K Hashimura nakatas@hsp.ncvc.go.jp 